\title{
Synthesis of Clustered Linear Arrays through a Total-Variation Compressive Sensing Approach
}

\author{
N. Anselmi, G. Gottardi, G. Oliveri, and A. Massa
}

\begin{abstract}
A new methodology for the synthesis of linear clustered arrays is presented in this work. More in detail, the design is carried out within the compressive sensing (CS) framework by formulating the problem at hand as the search of the feeding network weights with maximally-sparse gradient. Accordingly, the problem is solved thanks to a customized total-variation CS (TV-CS) method able to guarantee the matching of user-defined constraints on the radiated pattern and at the same time minimizing the number of clusters within the aperture. Some numerical results are shown to verify the effectiveness and the potentialities of the proposed methodology.
\end{abstract}


Contents

1 Numerical Results

2

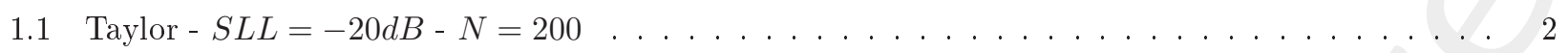

1.2 Taylor $-S L L=-20 d B-N=1000 \ldots \ldots \ldots \ldots \ldots \ldots \ldots \ldots \ldots \ldots \ldots$

page $1 / 18$ 


\section{Numerical Results}

1.1 Taylor $-S L L=-20 d B-N=200$

\section{Array Geometry:}

- Linear Array

- Number of Elements: $N=200$

- Element Spacing: $\Delta L_{R E F}=\lambda / 2$

- Aperture Length: $L=99.5 \lambda$

\section{Reference Pattern:}

- Pencil Beam, Taylor

- Number of elements: $N=200$

- Transition Index: $\bar{n}=6$

- Sidelobe Ratio: $S L L=-20 d B$

\section{Pareto Parameters:}

- Pattern Samples: $K \in\{4,6,8 \ldots, 20,25, \ldots, 50,60,70, \ldots, 100,300,400,500,1000\}$

- Primary penalty parameter: $\mu \in\left\{2 \times 10^{-2}, 2 \times 10^{-1}, \ldots, 2 \times 10^{13}\right\}$

- Secondary penalty parameter: $\beta \in\left\{2 \times 10^{-2}, 2 \times 10^{-1}, \ldots, 2 \times 10^{13}\right\}$

- $m_{t} \in\left\{1 \times 10^{1}, 2 \times 10^{1}, 5 \times 10^{1}, 1 \times 10^{2}, 5 \times 10^{2}, 1 \times 10^{3}, 2 \times 10^{3}\right\}$

- $m_{o} \in\left\{5 \times 10^{0}, 5 \times 10^{1}, 1 \times 10^{2}, 5 \times 10^{2}, 1 \times 10^{3}\right\}$

\section{TV-CS Parameters:}

- Starting primary penalty parameter: $\mu_{0}=\mu$ (default)

- Starting secondary penalty parameter: $\beta_{0}=\beta$ (default)

- Outer stopping tolerance: $t_{o}=1 \times 10^{-3}$ (default)

- Inner stopping tolerance: $t_{i}=1 \times 10^{-3}$ (default)

- Isotropic/anisotropic TV flag: $\mathcal{F}_{T V}=1$

- Negative/Positive signal: $\mathcal{F}_{N}=[$ false $]$ (default)

- TV $/$ L2 flag: $\mathcal{F}_{T 2}=[$ false $]$ (default) 
- Real/Imaginary signal flag: $\mathcal{F}_{R}=[$ false $]$ (default)

- Scaling Matrix A flag: $\mathcal{F}_{A}=[$ true $]$ (default)

- Scaling Vector B flag: $\mathcal{F}_{B}=[$ true $]$ (default)

- Guess Solution: $\mathcal{F}_{G}=0$ (all zeroes)

RESULTS - TOLERANCE: $\tau_{C}=1.0 \times 10^{-3}$

Pareto Front:

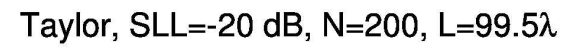

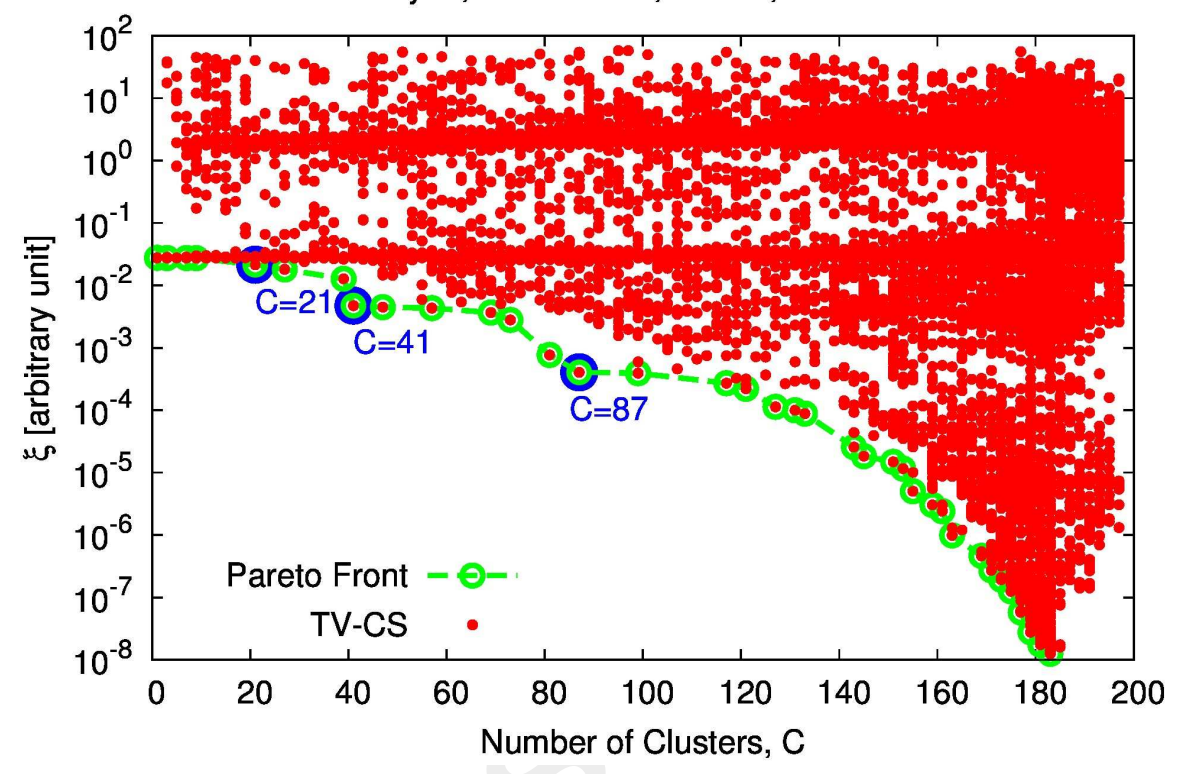

Figure 1: Performance Assessment (Taylor Pattern, $N=200, S L L=-20 \mathrm{~dB}, d=0.5 \lambda, L==99.5 \lambda$ )-Pareto front.

\begin{tabular}{|c|c|c|c|c|c|c|}
\hline$C$ & $\xi$ & $\mu$ & $\beta$ & $K$ & $m_{t}$ & $m_{o}$ \\
\hline 21 & $2.14 \times 10^{-2}$ & $2 \times 10^{-2}$ & $2 \times 10^{1}$ & 60 & $2 \times 10^{3}$ & $1 \times 10^{3}$ \\
\hline 41 & $4.73 \times 10^{-3}$ & $2 \times 10^{-2}$ & $2 \times 10^{1}$ & 80 & $2 \times 10^{3}$ & $1 \times 10^{3}$ \\
\hline 87 & $4.04 \times 10^{-4}$ & $2 \times 10^{-2}$ & $2 \times 10^{1}$ & 60 & $2 \times 10^{3}$ & $5 \times 10^{2}$ \\
\hline
\end{tabular}

Table I: Performance Assessment (Taylor Pattern, $N=200, S L L=-20 \mathrm{~dB}, d=0.5 \lambda, L=99.5 \lambda$ )-Selected solutions. 
Number of Clusters: $C=21$

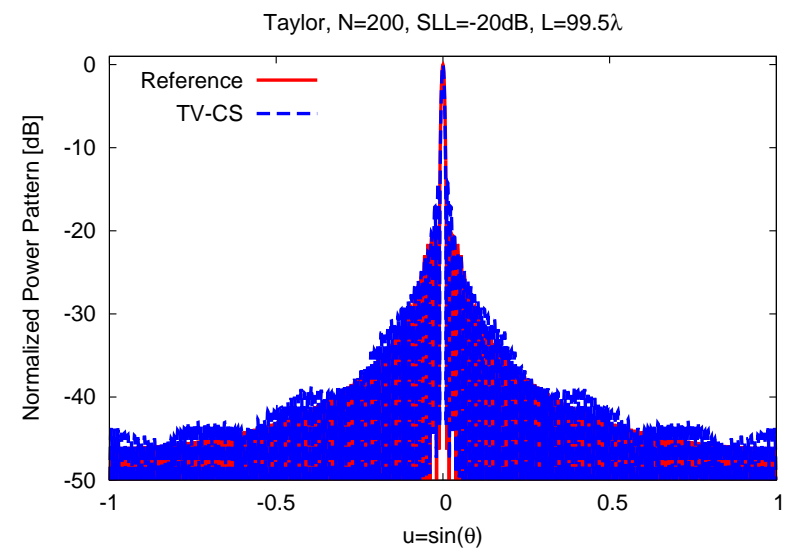

(a)

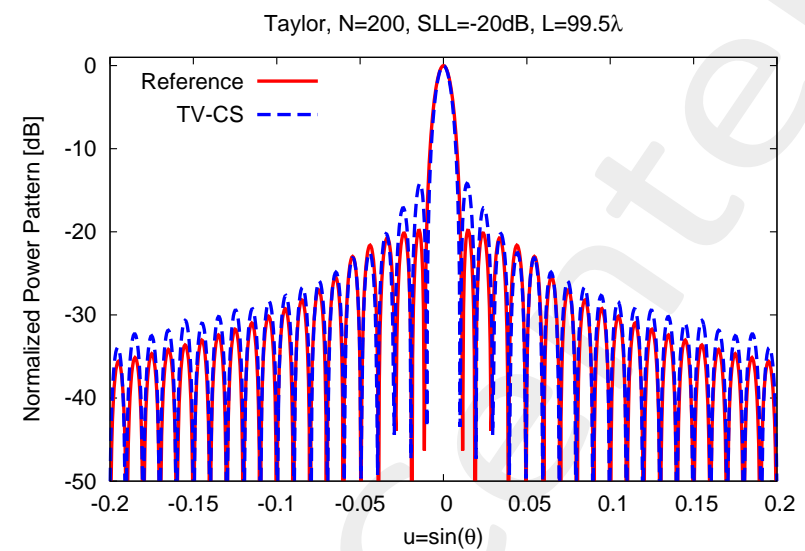

(b)

Figure 2: Performance Assessment (Taylor Pattern, $N=200, S L L=-20 \mathrm{~dB}, d=0.5 \lambda, L=99.5 \lambda, C=21$ )- Power pattern over the whole visible $u$-range (a) and a detail of the main lobe (b).

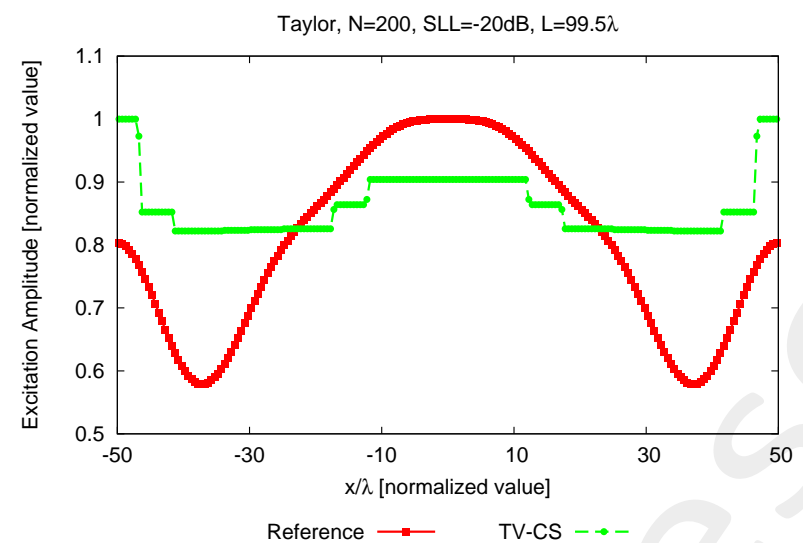

(a)

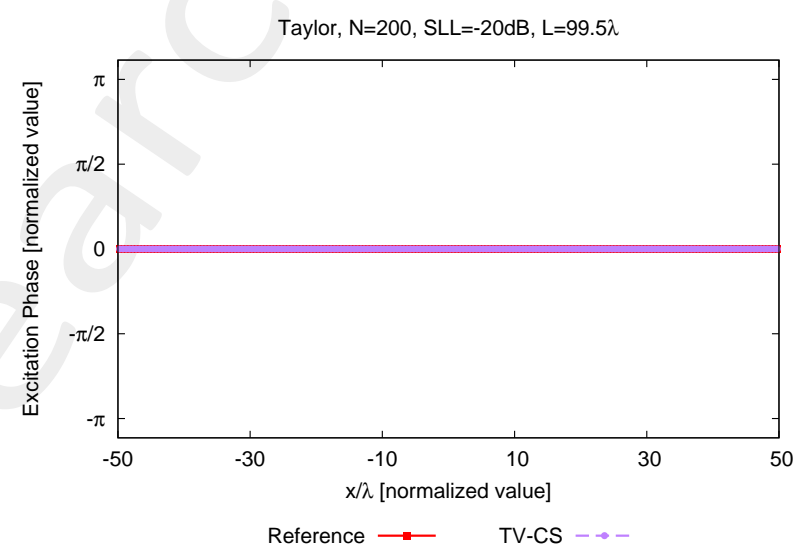

(b)

Figure 3: Performance Assessment (Taylor Pattern, $N=200, S L L=-20 \mathrm{~dB}, d=0.5 \lambda, L=99.5 \lambda, C=21$ )-Excitations amplitude (a) and phase (b).

Taylor, N=200, SLL=-20dB, L=99.5x

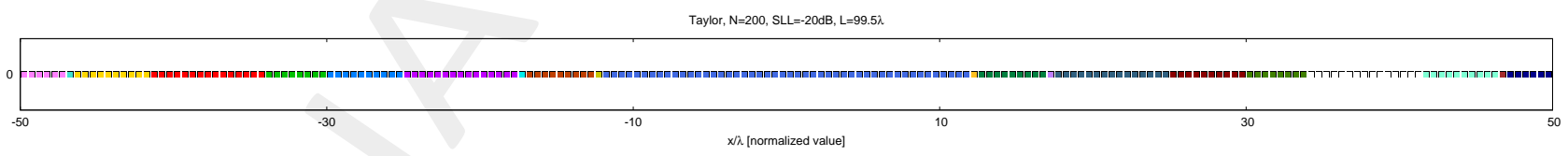

Figure 4: Performance Assessment (Taylor Pattern, $N=200, S L L=-20 \mathrm{~dB}, d=0.5 \lambda, L=99.5 \lambda, C=21$ )- Array elements clustering configuration.

\begin{tabular}{|c|c|c|c|c|c|c|}
\hline & $C$ & $S L L[\mathrm{~dB}]$ & $B W[\mathrm{deg}]$ & $D_{\max }[\mathrm{dB}]$ & $D R R_{\max }[\mathrm{dB}]$ & $\xi\left[10^{-2}\right.$ \\
\hline \hline Reference & - & -19.82 & 0.5421 & 22.87 & 2.37 & - \\
\hline$T V-C S$ & 21 & -14.19 & 0.5055 & 23.00 & 0.85 & 2.14 \\
\hline
\end{tabular}

Table II: Performance Assessment (Taylor Pattern, $N=200, S L L=-20 \mathrm{~dB}, d=0.5 \lambda, L=99.5 \lambda, C=21$ )- Array Performance Indexes. 
Number of Clusters: $C=41$

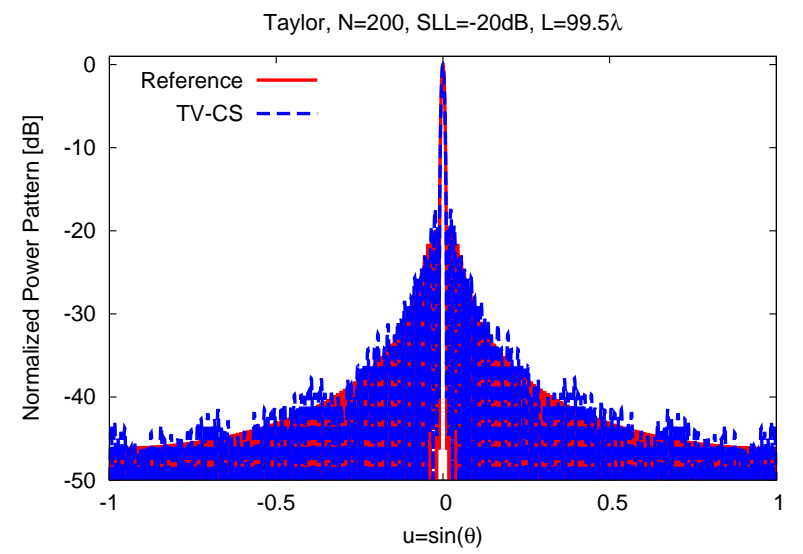

(a)

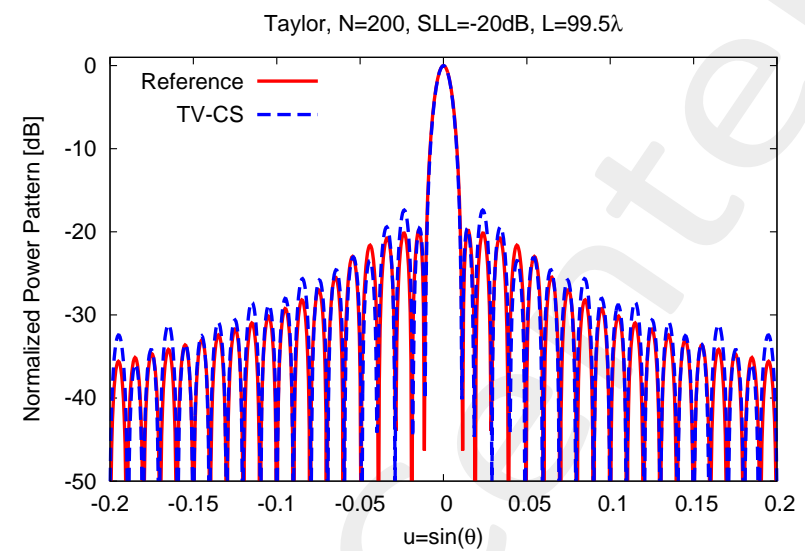

(b)

Figure 5: Performance Assessment (Taylor Pattern, $N=200, S L L=-20 \mathrm{~dB}, d=0.5 \lambda, L=99.5 \lambda, C=41$ )- Power pattern over the whole visible $u$-range (a) and a detail of the main lobe (b).

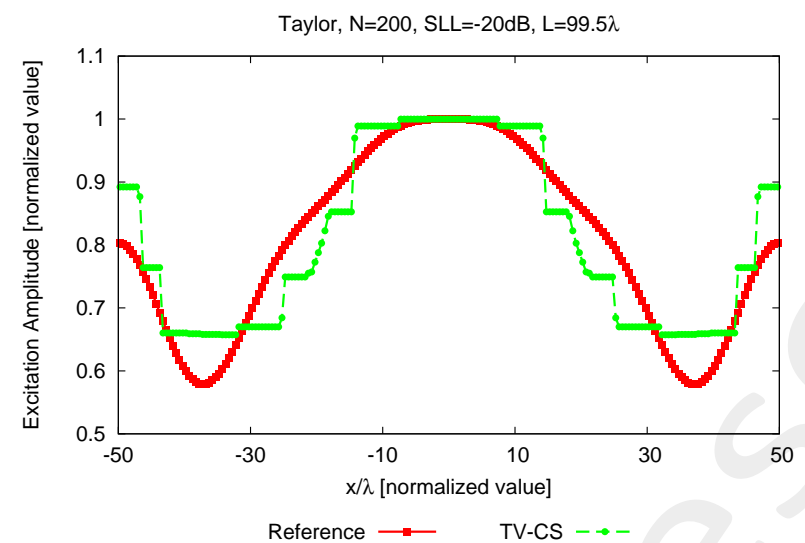

(a)

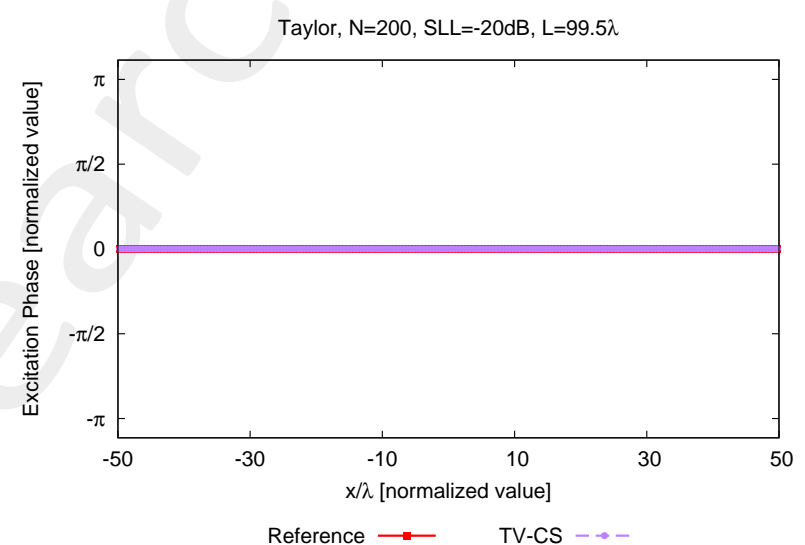

$(b)$

Figure 6: Performance Assessment (Taylor Pattern, $N=200, S L L=-20 \mathrm{~dB}, d=0.5 \lambda, L=99.5 \lambda, C=41$ )-Excitations amplitude (a) and phase (b).

Taylor, N=200, SLL=-20dB, L=99.5

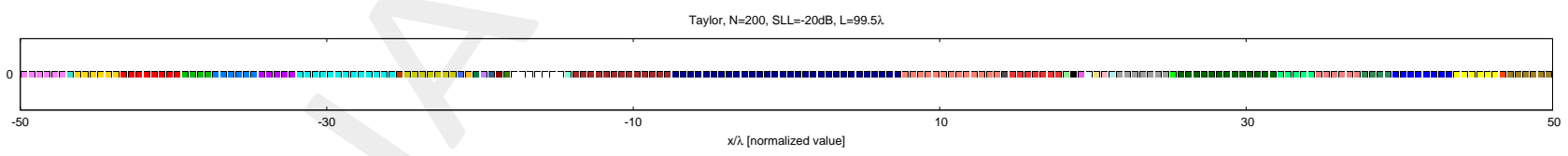

Figure 7: Performance Assessment (Taylor Pattern, $N=200, S L L=-20 \mathrm{~dB}, d=0.5 \lambda, L=99.5 \lambda, C=41$ )- Array elements clustering configuration.

\begin{tabular}{|c|c|c|c|c|c|c|}
\hline+ & $C$ & $S L L[\mathrm{~dB}]$ & $B W[\operatorname{deg}]$ & $D_{\max }[\mathrm{dB}]$ & $D R R_{\max }[\mathrm{dB}]$ & \begin{tabular}{l|l}
$\xi$ & $\times 10^{-3}$ \\
\end{tabular} \\
\hline Reference & - & -19.82 & 0.5421 & 22.87 & 2.37 & - \\
\hline$T V-C S$ & 41 & -17.37 & 0.5317 & 22.89 & 1.82 & 4.73 \\
\hline
\end{tabular}

Table III: Performance Assessment (Taylor Pattern, $N=200, S L L=-20 \mathrm{~dB}, d=0.5 \lambda, L=99.5 \lambda, C=41$ )- Array Performance Indexes. 
Number of Clusters: $C=87$

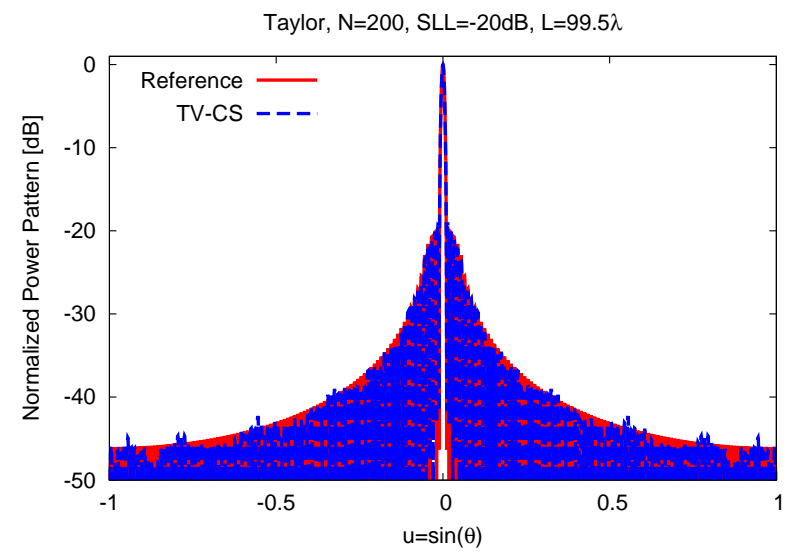

(a)

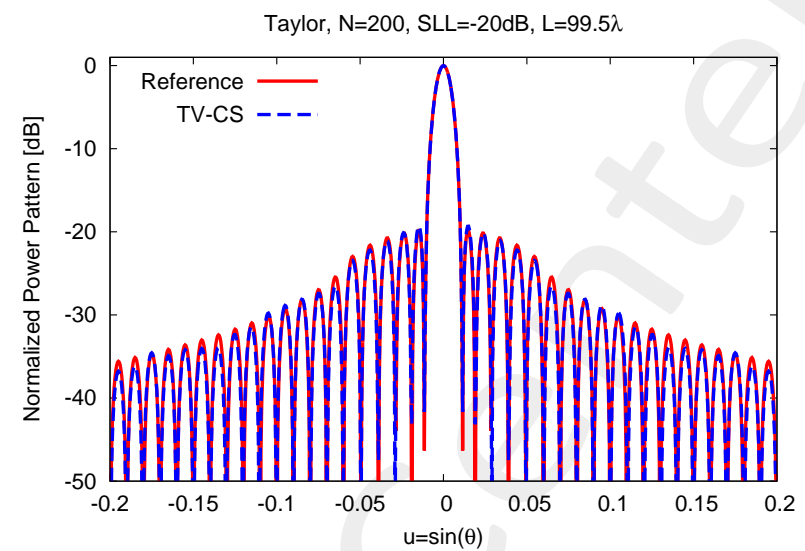

(b)

Figure 8: Performance Assessment (Taylor Pattern, $N=200, S L L=-20 \mathrm{~dB}, d=0.5 \lambda, L=99.5 \lambda, C=87$ )-Power pattern over the whole visible $u$-range (a) and a detail of the main lobe (b).

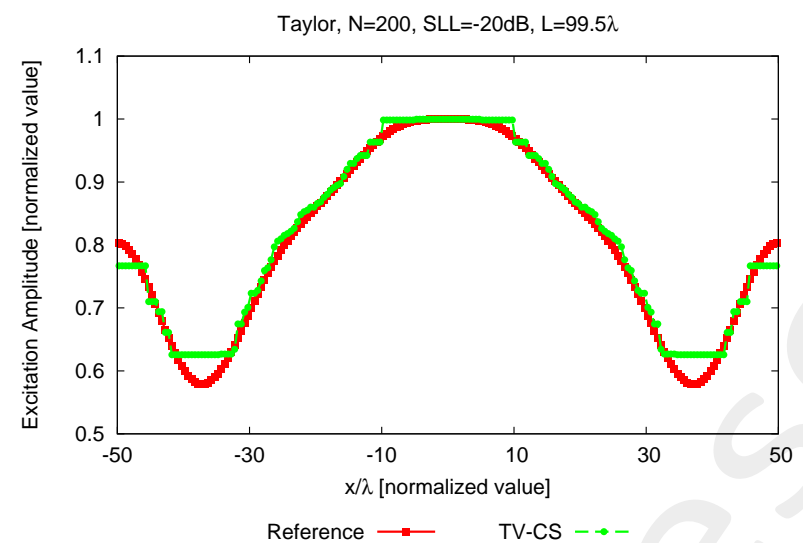

(a)

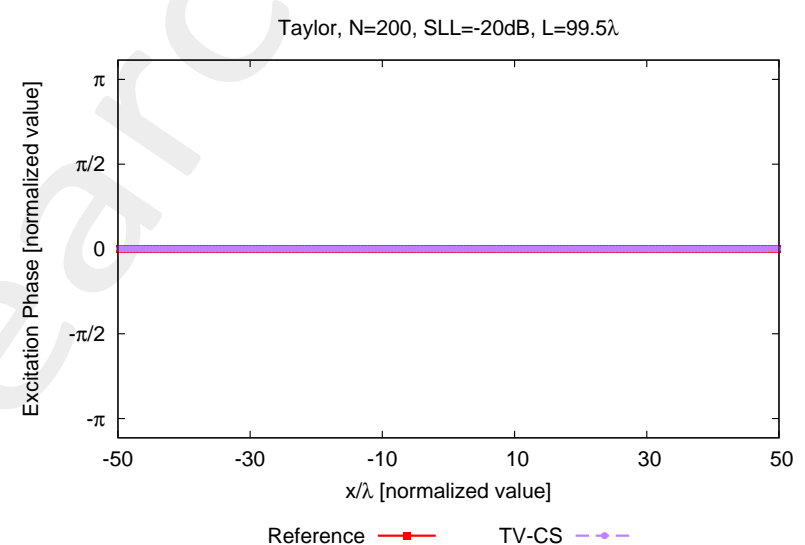

$(b)$

Figure 9: Performance Assessment (Taylor Pattern, $N=200, S L L=-20 \mathrm{~dB}, d=0.5 \lambda, L=99.5 \lambda, C=87$ )-Excitations amplitude (a) and phase (b).

Taylor, N=200, SLL=-20dB, L=99.5x

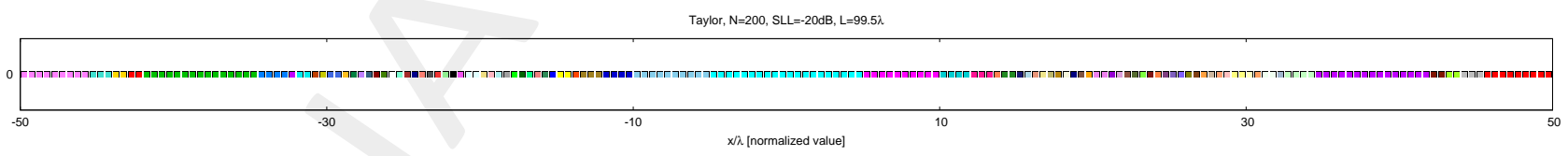

Figure 10: Performance Assessment (Taylor Pattern, $N=200, S L L=-20 \mathrm{~dB}, d=0.5 \lambda, L=99.5 \lambda, C=87$ )- Array elements clustering configuration.

\begin{tabular}{|c|c|c|c|c|c|c|}
\hline+ & $C$ & $S L L[\mathrm{~dB}]$ & $B W[\operatorname{deg}]$ & $D_{\max }[\mathrm{dB}]$ & $D R R_{\max }[\mathrm{dB}]$ & \begin{tabular}{l|l}
$\xi$ & $\times 10^{-4}$ \\
\end{tabular} \\
\hline Reference & - & -19.82 & 0.5421 & 22.87 & 2.37 & - \\
\hline$T V-C S$ & 87 & -19.33 & 0.5421 & 22.89 & 2.04 & 4.04 \\
\hline
\end{tabular}

Table IV: Performance Assessment (Taylor Pattern, $N=200, S L L=-20 \mathrm{~dB}, d=0.5 \lambda, L=99.5 \lambda, C=87$ )- Array Performance Indexes. 
RESULTS - TOLERANCE: $\tau_{C}=1.0 \times 10^{-2}$

NOTE: With respect to the previous test cases the clustering "de-noise" tolerance has been decreased from $\tau_{c}=1 \times 10^{-3}$ to $\tau_{c}=1 \times 10^{-2}$.

Pareto Front:

Taylor, SLL=-20 dB, N=200, L=99.5 $\lambda$

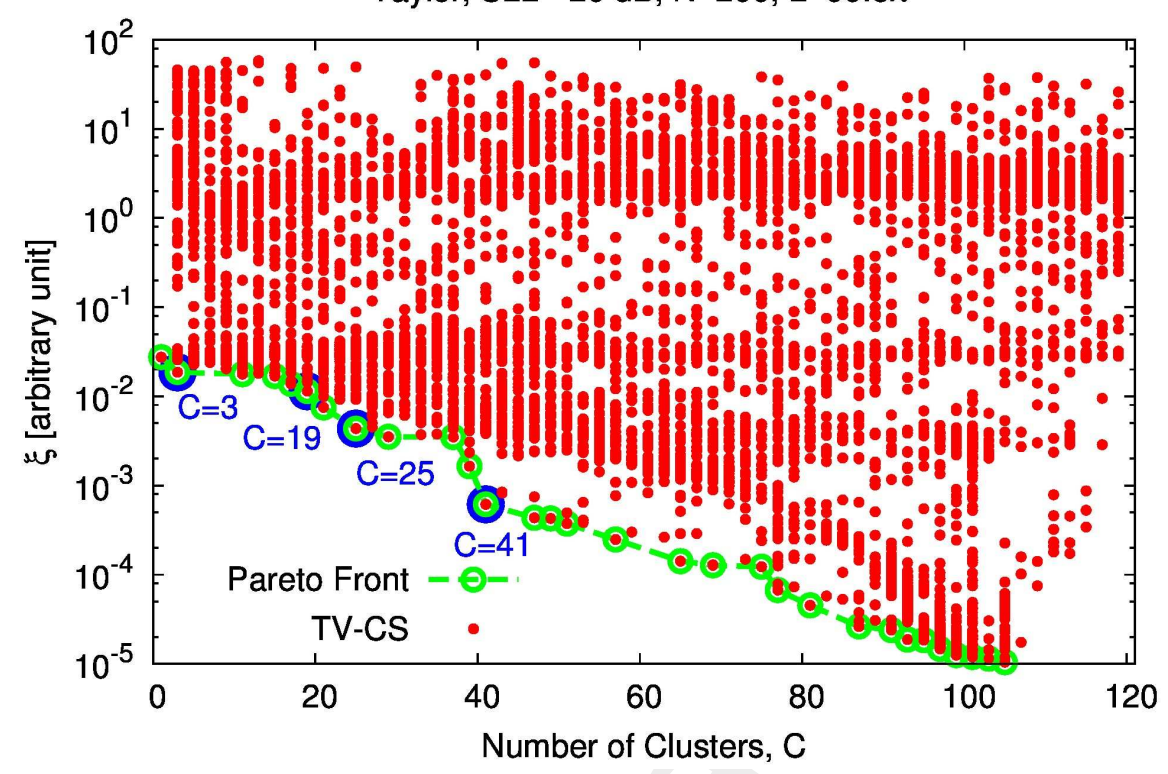

Figure 11: Performance Assessment (Taylor Pattern, $N=200, S L L=-20 \mathrm{~dB}, d=0.5 \lambda, L==99.5 \lambda$ ) - Pareto front.

\begin{tabular}{|c|c|c|c|c|c|c|}
\hline$C$ & $\xi$ & $\mu$ & $\beta$ & $K$ & $m_{t}$ & $m_{o}$ \\
\hline 3 & $1.86 \times 10^{-2}$ & $2 \times 10^{-2}$ & $2 \times 10^{1}$ & 40 & $2 \times 10^{3}$ & $1 \times 10^{3}$ \\
\hline 19 & $1.14 \times 10^{-2}$ & $2 \times 10^{-2}$ & $2 \times 10^{3}$ & 1000 & $5 \times 10^{2}$ & $5 \times 10^{1}$ \\
\hline 25 & $4.36 \times 10^{-3}$ & $2 \times 10^{-2}$ & $2 \times 10^{1}$ & 500 & $1 \times 10^{3}$ & $1 \times 10^{3}$ \\
\hline 25 & $6.16 \times 10^{-4}$ & $2 \times 10^{-2}$ & $2 \times 10^{1}$ & 1000 & $1 \times 10^{3}$ & $5 \times 10^{2}$ \\
\hline
\end{tabular}

Table V: Performance Assessment (Taylor Pattern, $N=200, S L L=-20 \mathrm{~dB}, d=0.5 \lambda, L=99.5 \lambda$ )-Selected solutions. 
Number of Clusters: $C=3$

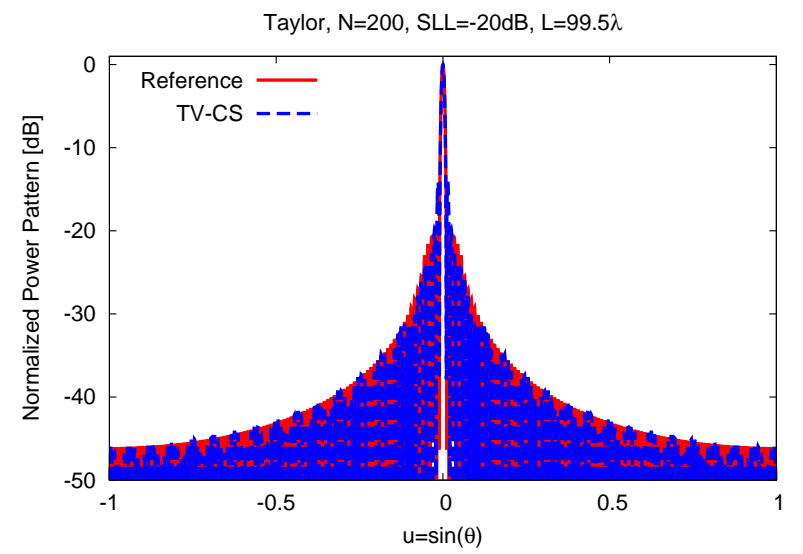

(a)

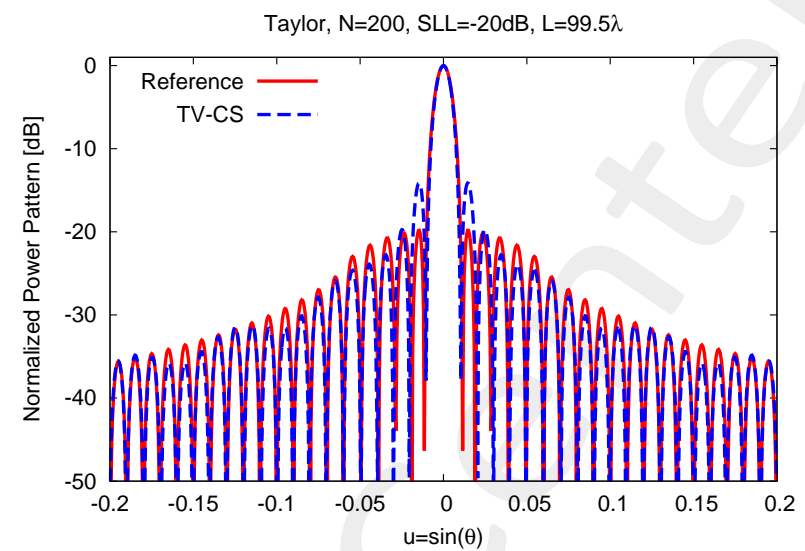

(b)

Figure 12: Performance Assessment (Taylor Pattern, $N=200, S L L=-20 \mathrm{~dB}, d=0.5 \lambda, L=99.5 \lambda, C=3$ )-Power pattern over the whole visible $u$-range (a) and a detail of the main lobe (b).

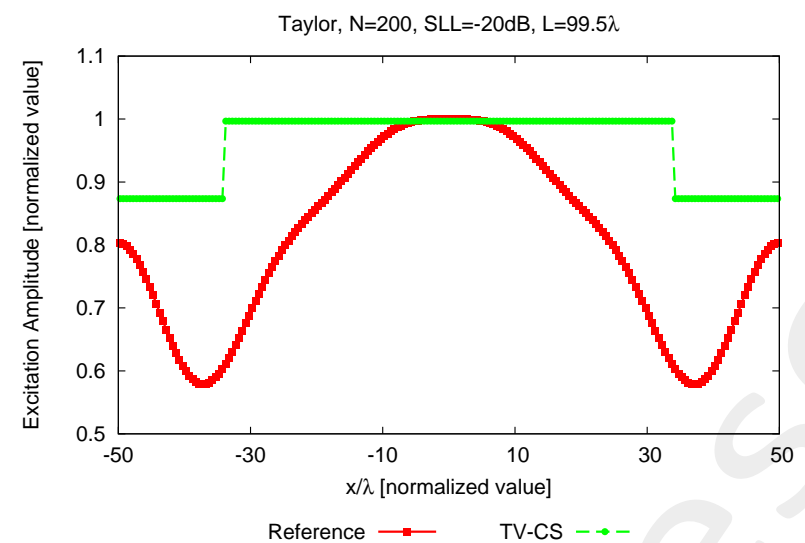

(a)

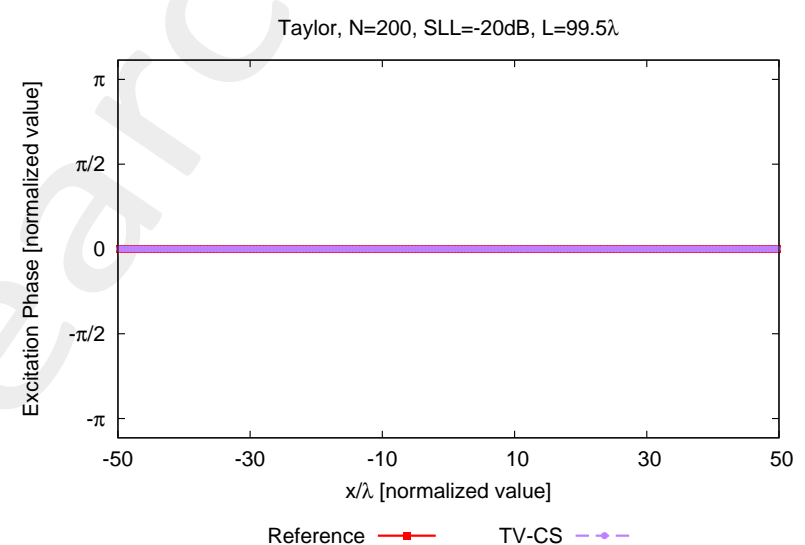

$(b)$

Figure 13: Performance Assessment (Taylor Pattern, $N=200, S L L=-20 \mathrm{~dB}, d=0.5 \lambda, L=99.5 \lambda, C=3$ )-Excitations amplitude (a) and phase (b).

Taylor, N=200, SLL=-20dB, L=99.5

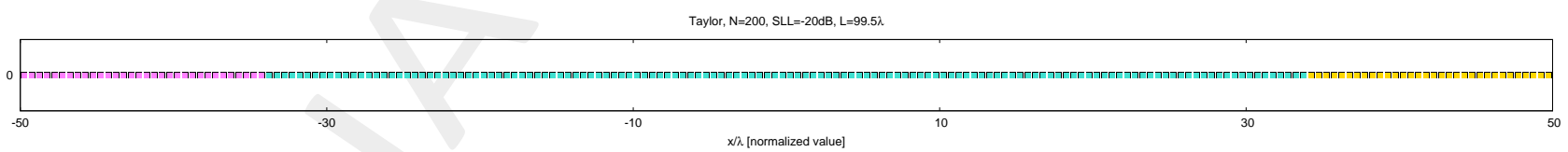

Figure 14: Performance Assessment (Taylor Pattern, $N=200, S L L=-20 \mathrm{~dB}, d=0.5 \lambda, L=99.5 \lambda, C=3$ )- Array elements clustering configuration.

\begin{tabular}{|c|c|c|c|c|c|c|}
\hline & $C$ & $S L L[\mathrm{~dB}]$ & $B W[\mathrm{deg}]$ & $D_{\max }[\mathrm{dB}]$ & $D R R_{\max }[\mathrm{dB}]$ & $\xi\left[10^{-2}\right]$ \\
\hline \hline Reference & - & -19.82 & 0.5421 & 22.87 & 2.37 & - \\
\hline$T V-C S$ & 3 & -14.13 & 0.5196 & 22.99 & 0.57 & 1.86 \\
\hline
\end{tabular}

Table VI: Performance Assessment (Taylor Pattern, $N=200, S L L=-20 \mathrm{~dB}, d=0.5 \lambda, L=99.5 \lambda, C=3$ )- Array Performance Indexes. 
Number of Clusters: $C=19$

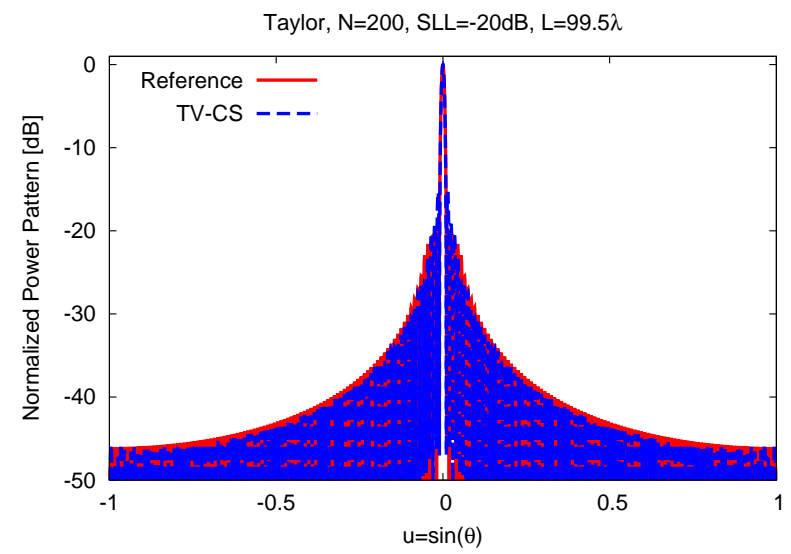

(a)

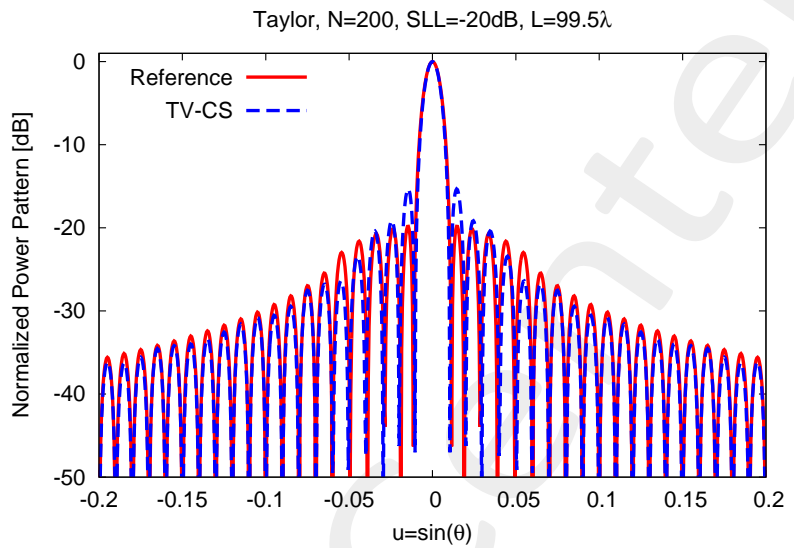

(b)

Figure 15: Performance Assessment (Taylor Pattern, $N=200, S L L=-20 \mathrm{~dB}, d=0.5 \lambda, L=99.5 \lambda, C=19$ )- Power pattern over the whole visible $u$-range (a) and a detail of the main lobe (b).

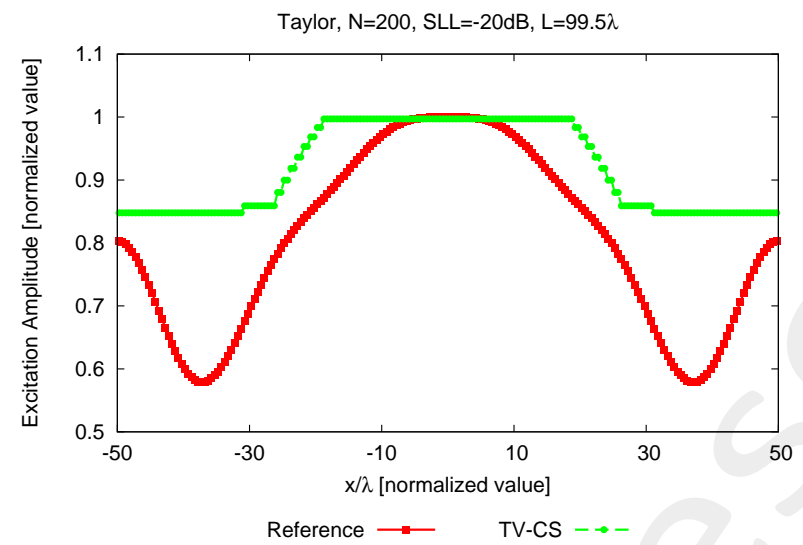

(a)

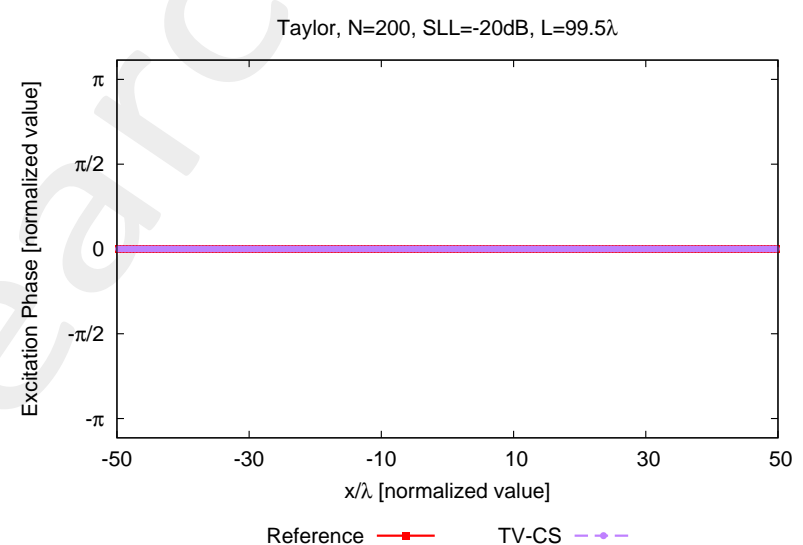

$(b)$

Figure 16: Performance Assessment (Taylor Pattern, $N=200, S L L=-20 \mathrm{~dB}, d=0.5 \lambda, L=99.5 \lambda, C=19$ )-Excitations amplitude (a) and phase (b).

Taylor, N=200, SLL=-20dB, L=99.5

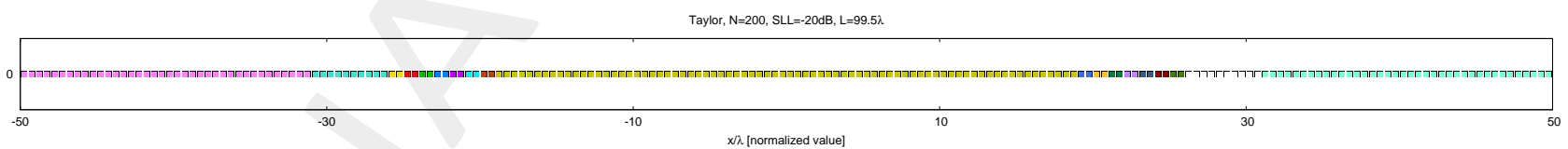

Figure 17: Performance Assessment (Taylor Pattern, $N=200, S L L=-20 \mathrm{~dB}, d=0.5 \lambda, L=99.5 \lambda, C=19$ )- Array elements clustering configuration.

\begin{tabular}{|c|c|c|c|c|c|c|}
\hline+ & $C$ & $S L L[\mathrm{~dB}]$ & $B W[\operatorname{deg}]$ & $D_{\max }[\mathrm{dB}]$ & $D R R_{\max }[\mathrm{dB}]$ & \begin{tabular}{l|l}
$\xi$ & $\times 10^{-2}$ \\
\end{tabular} \\
\hline Reference & - & -19.82 & 0.5421 & 22.87 & 2.37 & - \\
\hline$T V-C S$ & 19 & -15.33 & 0.5232 & 22.99 & 0.70 & 1.14 \\
\hline
\end{tabular}

Table VII: Performance Assessment (Taylor Pattern, $N=200, S L L=-20 \mathrm{~dB}, d=0.5 \lambda, L=99.5 \lambda, C=19$ )- Array Performance Indexes. 
Number of Clusters: $C=25$

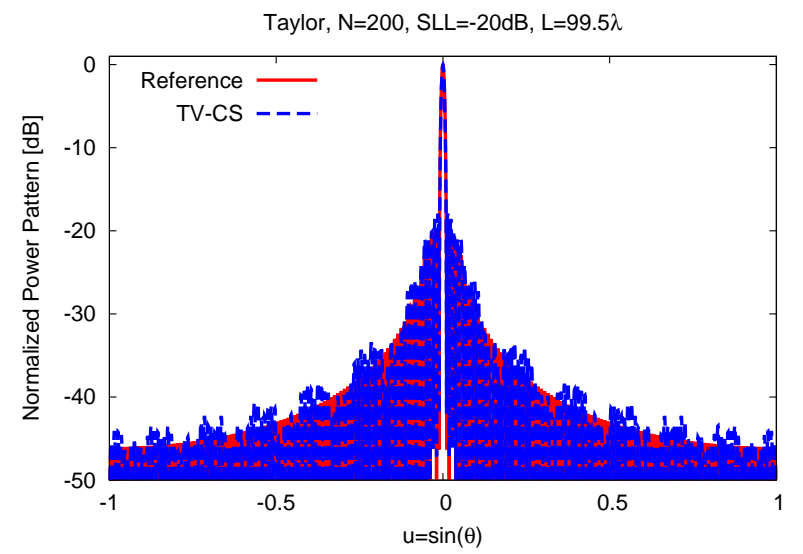

(a)

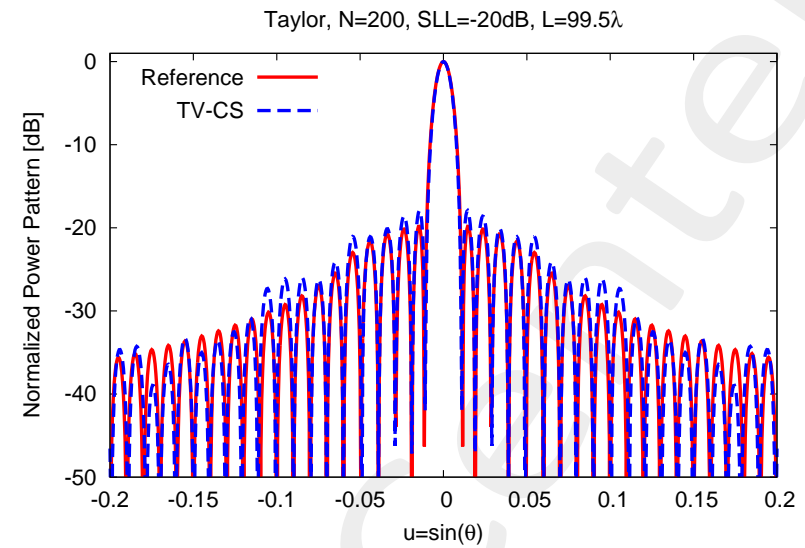

(b)

Figure 18: Performance Assessment (Taylor Pattern, $N=200, S L L=-20 \mathrm{~dB}, d=0.5 \lambda, L=99.5 \lambda, C=25$ )-Power pattern over the whole visible $u$-range (a) and a detail of the main lobe (b).

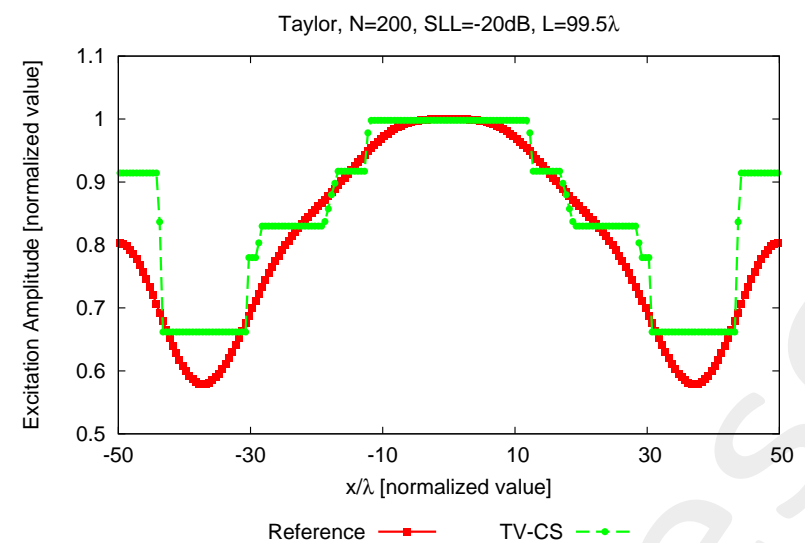

(a)

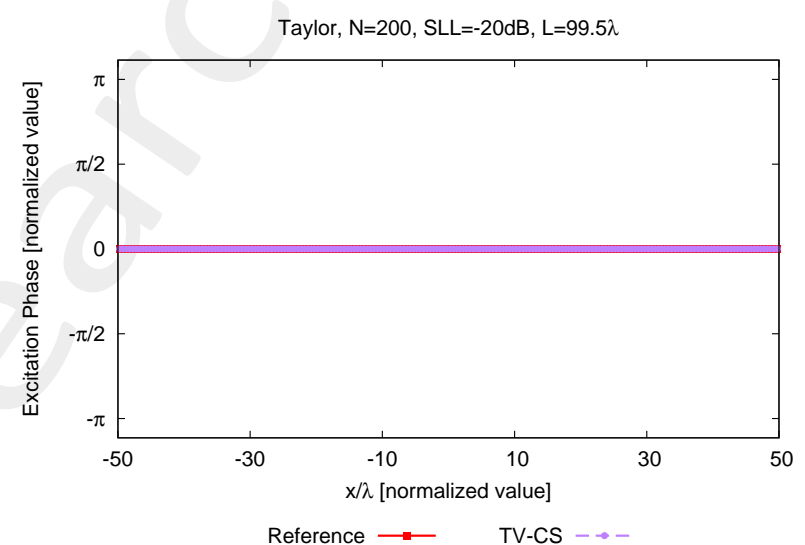

$(b)$

Figure 19: Performance Assessment (Taylor Pattern, $N=200, S L L=-20 \mathrm{~dB}, d=0.5 \lambda, L=99.5 \lambda, C=25$ )-Excitations amplitude (a) and phase (b).

Taylor, N=200, SLL=-20dB, L=99.5

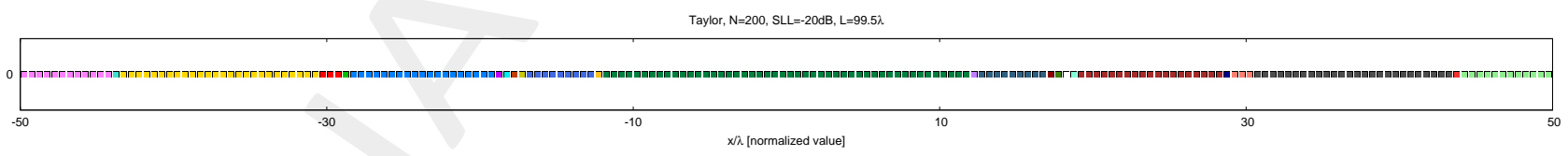

Figure 20: Performance Assessment (Taylor Pattern, $N=200, S L L=-20 \mathrm{~dB}, d=0.5 \lambda, L=99.5 \lambda, C=25$ )- Array elements clustering configuration.

\begin{tabular}{|c|c|c|c|c|c|c|}
\hline & $C$ & $S L L[\mathrm{~dB}]$ & $B W$ [deg] & $D_{\max }[\mathrm{dB}]$ & $D R R_{\max }[\mathrm{dB}]$ & \begin{tabular}{l|l}
$\xi$ & $\times 10^{-3}$ \\
\end{tabular} \\
\hline Reference & - & -19.82 & 0.5421 & 22.87 & 2.37 & - \\
\hline$T V-C S$ & 25 & -17.93 & 0.5270 & 22.92 & 1.78 & 4.36 \\
\hline
\end{tabular}

Table VIII: Performance Assessment (Taylor Pattern, $N=200, S L L=-20 \mathrm{~dB}, d=0.5 \lambda, L=99.5 \lambda$, $C=25)$ - Array Performance Indexes. 
Number of Clusters: $C=41$

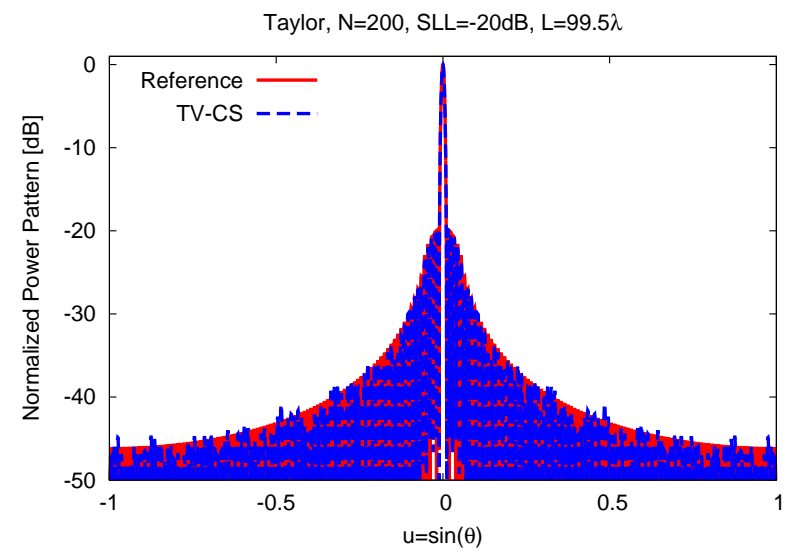

(a)

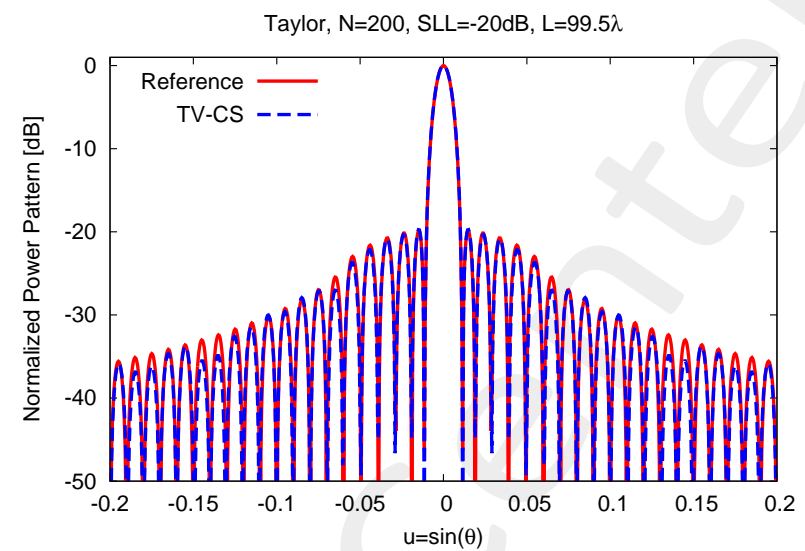

(b)

Figure 21: Performance Assessment (Taylor Pattern, $N=200, S L L=-20 \mathrm{~dB}, d=0.5 \lambda, L=99.5 \lambda, C=41$ )- Power pattern over the whole visible $u$-range (a) and a detail of the main lobe (b).

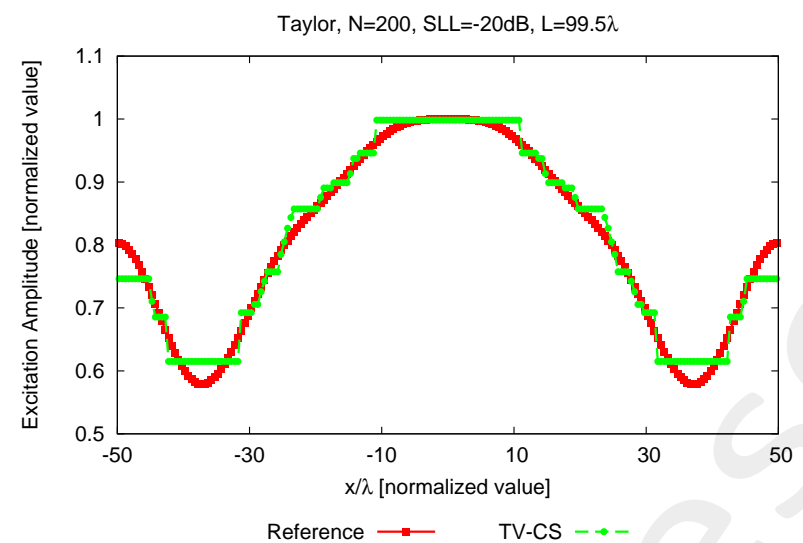

(a)

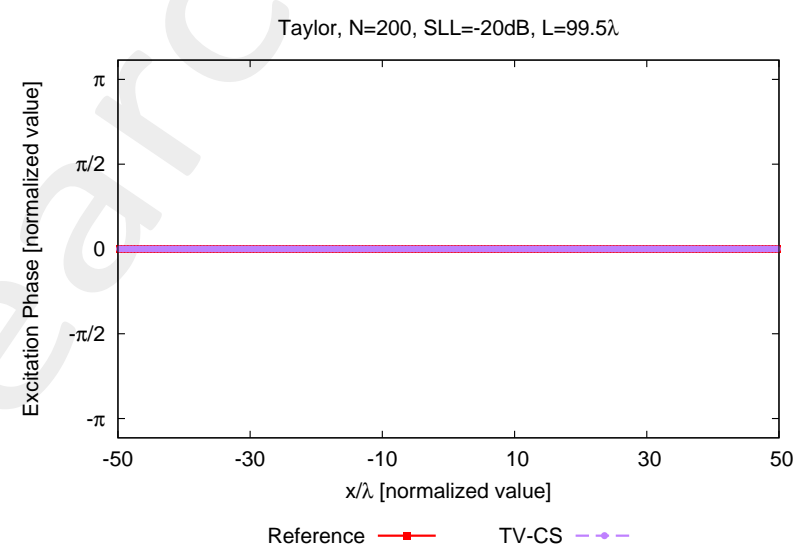

$(b)$

Figure 22: Performance Assessment (Taylor Pattern, $N=200, S L L=-20 \mathrm{~dB}, d=0.5 \lambda, L=99.5 \lambda, C=41$ )-Excitations amplitude (a) and phase (b).

Taylor, N=200, SLL=-20dB, L=99.5

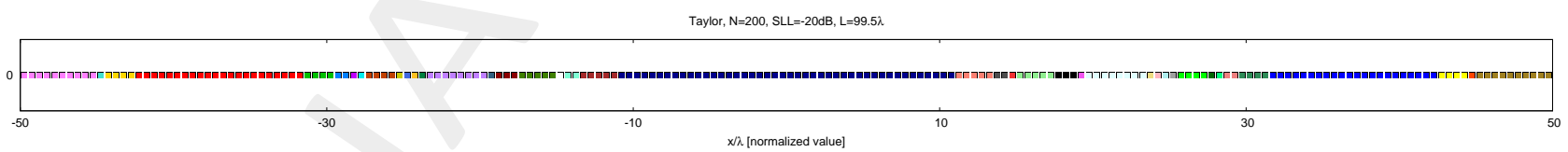

Figure 23: Performance Assessment (Taylor Pattern, $N=200, S L L=-20 \mathrm{~dB}, d=0.5 \lambda, L=99.5 \lambda, C=41$ )- Array elements clustering configuration.

\begin{tabular}{|c|c|c|c|c|c|c|}
\hline & $C$ & $S L L[\mathrm{~dB}]$ & $B W$ [deg] & $D_{\max }[\mathrm{dB}]$ & $D R R_{\max }[\mathrm{dB}]$ & \begin{tabular}{l|l}
$\xi$ & $\times 10^{-4}$ \\
\end{tabular} \\
\hline Reference & - & -19.82 & 0.5421 & 22.87 & 2.37 & - \\
\hline$T V-C S$ & 41 & -19.74 & 0.5445 & 22.88 & 2.10 & 6.16 \\
\hline
\end{tabular}

Table IX: Performance Assessment (Taylor Pattern, $N=200, S L L=-20 \mathrm{~dB}, d=0.5 \lambda, L=99.5 \lambda, C=41$ )- Array Performance Indexes. 
1.2 Taylor $-S L L=-20 d B-N=1000$

\section{Array Geometry:}

- Linear Array

- Number of Elements: $N=1000$

- Element Spacing: $\Delta L_{R E F}=\lambda / 2$

- Aperture Length: $L=499.5 \lambda$

\section{Reference Pattern:}

- Pencil Beam, Taylor

- Number of elements: $N=1000$

- Transition Index: $\bar{n}=6$

- Sidelobe Ratio: $S L L=-20 d B$

\section{Pareto Parameters:}

- Pattern Samples: $K \in\{4,6,8 \ldots, 20,25, \ldots, 50,60,70, \ldots, 100,300,400,500,1000\}$

- Primary penalty parameter: $\mu \in\left\{2 \times 10^{-2}, 2 \times 10^{-1}, \ldots, 2 \times 10^{13}\right\}$

- Secondary penalty parameter: $\beta \in\left\{2 \times 10^{-2}, 2 \times 10^{-1}, \ldots, 2 \times 10^{13}\right\}$

- $m_{t} \in\left\{1 \times 10^{1}, 2 \times 10^{1}, 5 \times 10^{1}, 1 \times 10^{2}, 5 \times 10^{2}, 1 \times 10^{3}\right\}$

\section{TV-CS Parameters:}

- Starting primary penalty parameter: $\mu_{0}=\mu$ (default)

- Starting secondary penalty parameter: $\beta_{0}=\beta$ (default)

- Outer stopping tolerance: $t_{o}=1 \times 10^{-3}$ (default)

- Inner stopping tolerance: $t_{i}=1 \times 10^{-3}$ (default)

- Outer maximum iterations: $m_{o}=10$ (default)

- Isotropic/anisotropic TV flag: $\mathcal{F}_{T V}=1$

- Negative/Positive signal: $\mathcal{F}_{N}=[$ false $]$ (default)

- TV $/ \mathrm{L} 2$ flag: $\mathcal{F}_{T 2}=[$ false $]$ (default)

- Real/Imaginary signal flag: $\mathcal{F}_{R}=[$ false $]$ (default) 
- Scaling Matrix A flag: $\mathcal{F}_{A}=[$ true $]$ (default)

- Scaling Vector B flag: $\mathcal{F}_{B}=[$ true $]$ (default)

- Guess Solution: $\mathcal{F}_{G}=0$ (all zeroes)

RESULTS - TOLERANCE: $\tau_{C}=1.0 \times 10^{-2}$

Pareto Front:

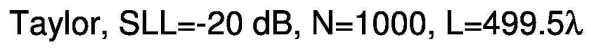

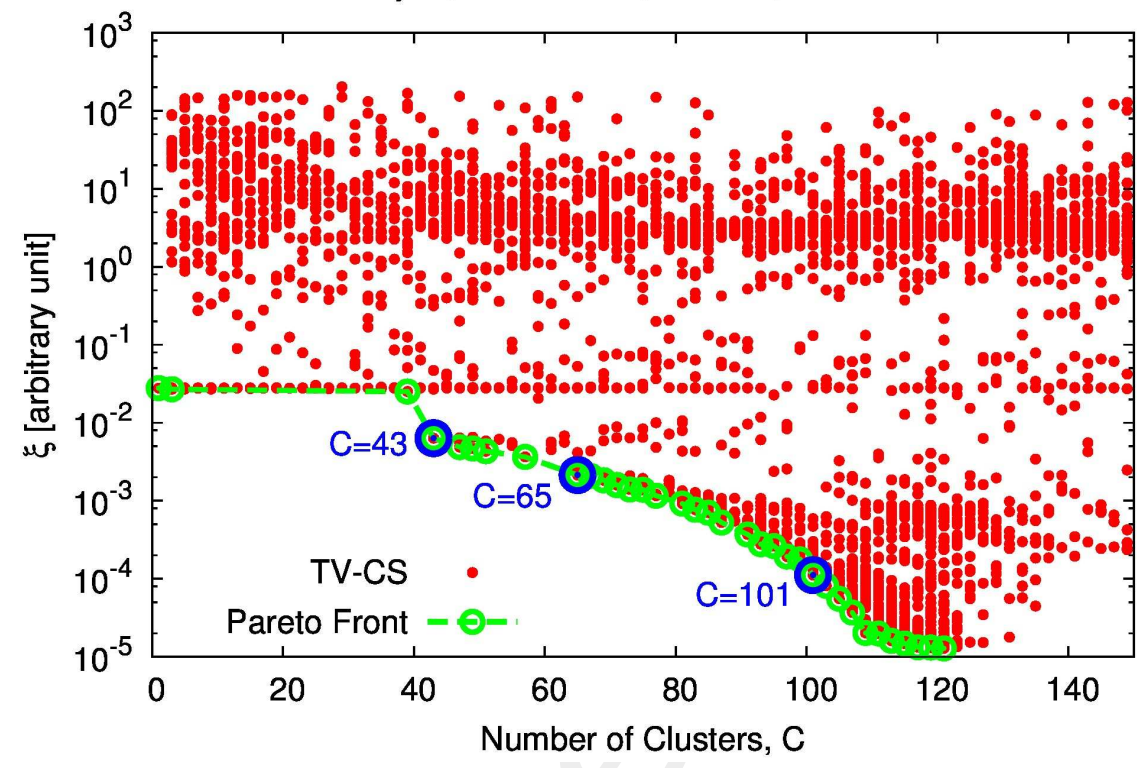

Figure 24: Performance Assessment (Taylor Pattern, $N=1000, S L L=-20 \mathrm{~dB}, d=0.5 \lambda, L=499.5 \lambda$ )-Pareto front.

\begin{tabular}{|c|c|c|c|c|c|c|}
\hline$C$ & $\xi$ & $\mu$ & $\beta$ & $K$ & $m_{t}$ & $m_{o}$ \\
\hline 43 & $6.35 \times 10^{-3}$ & $2 \times 10^{-2}$ & $2 \times 10^{2}$ & $1 \times 10^{3}$ & $1 \times 10^{3}$ & $1 \times 10^{2}$ \\
\hline 65 & $2.14 \times 10^{-3}$ & $2 \times 10^{-1}$ & $2 \times 10^{+6}$ & $1 \times 10^{3}$ & $5 \times 10^{2}$ & $1 \times 10^{2}$ \\
\hline 101 & $1.12 \times 10^{-4}$ & $2 \times 10^{-2}$ & $2 \times 10^{3}$ & $5 \times 10^{3}$ & $5 \times 10^{2}$ & $5 \times 10^{1}$ \\
\hline
\end{tabular}

Table X: Performance Assessment (Taylor Pattern, $N=1000, S L L=-20 \mathrm{~dB}, d=0.5 \lambda, L=499.5 \lambda$ )-Selected solutions. 
Number of Clusters: $C=43$

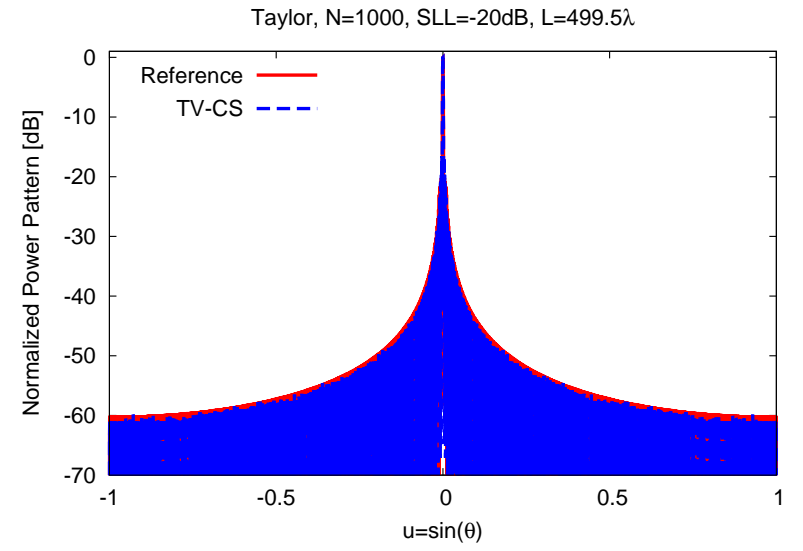

(a)

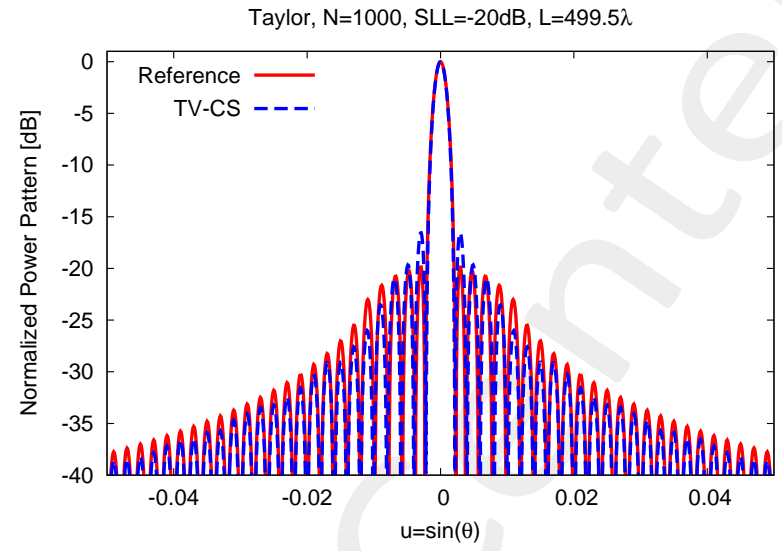

(b)

Figure 25: Performance Assessment (Taylor Pattern, $N=1000, S L L=-20 \mathrm{~dB}, d=0.5 \lambda, L=499.5 \lambda$, $C=43)$ - Power pattern over the whole visible $u$-range (a) and a detail of the main lobe (b).

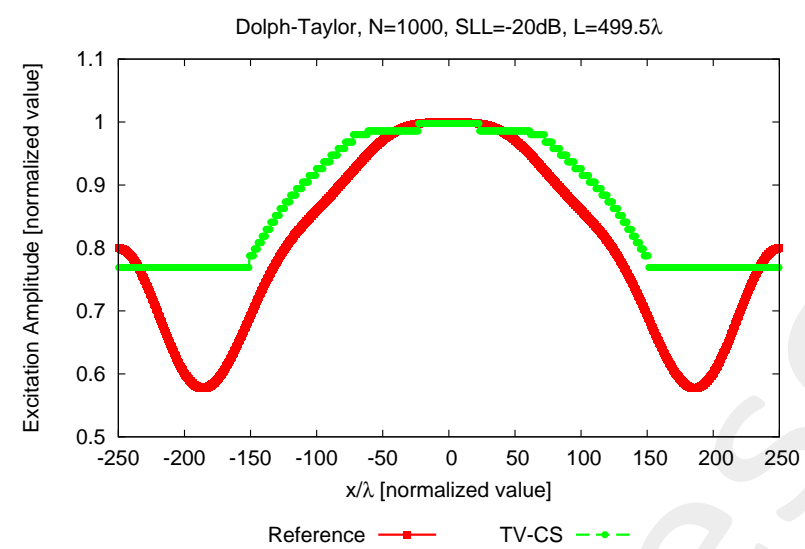

(a)

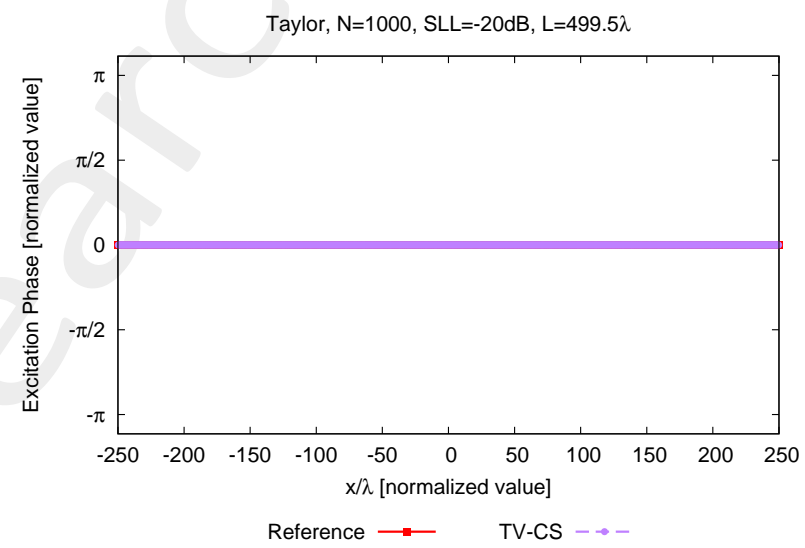

(b)

Figure 26: Performance Assessment (Taylor Pattern, $N=1000, S L L=-20 \mathrm{~dB}, d=0.5 \lambda, L=499.5 \lambda$, $C=43)$-Excitations amplitude (a) and phase (b).

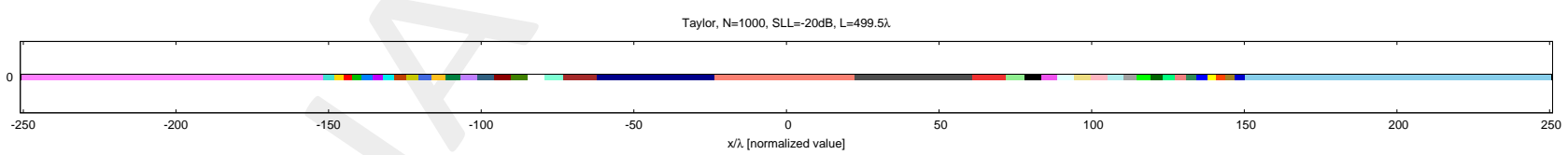

Figure 27: Performance Assessment (Taylor Pattern, $N=1000, S L L=-20 \mathrm{~dB}, d=0.5 \lambda, L=499.5 \lambda$, $C=43$ )-Array elements clustering configuration.

\begin{tabular}{|c|c|c|c|c|c|c|}
\hline & $C$ & $S L L[\mathrm{~dB}]$ & $B W[\mathrm{deg}]$ & $D_{\max }[\mathrm{dB}]$ & $D R R_{\max }[\mathrm{dB}]$ & $\xi\left[\times 10^{-3}\right]$ \\
\hline \hline Reference & - & -19.87 & 0.1085 & 29.86 & 2.38 & - \\
\hline$T V-C S$ & 43 & -16.56 & 1.0640 & 29.95 & 1.13 & 6.35 \\
\hline
\end{tabular}

Table XI: Performance Assessment (Taylor Pattern, $N=1000, S L L=-20 \mathrm{~dB}, d=0.5 \lambda, L=499.5 \lambda$, $C=3$ )-Array Performance Indexes. 
Number of Clusters: $C=65$

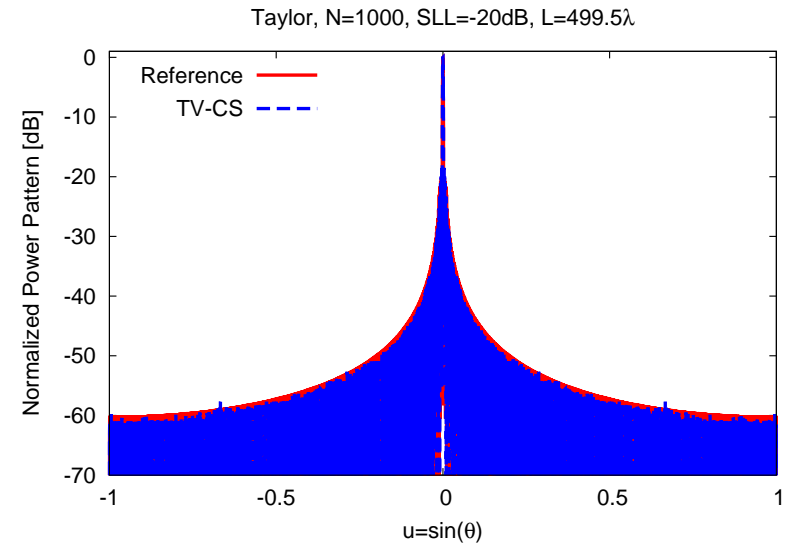

(a)

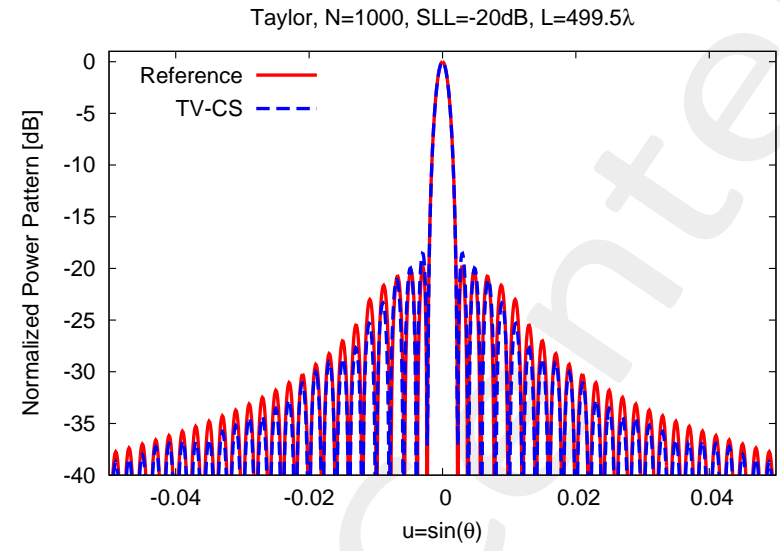

(b)

Figure 28: Performance Assessment (Taylor Pattern, $N=1000, S L L=-20 \mathrm{~dB}, d=0.5 \lambda, L=499.5 \lambda$, $C=65$ )-Power pattern over the whole visible $u$-range (a) and a detail of the main lobe (b).

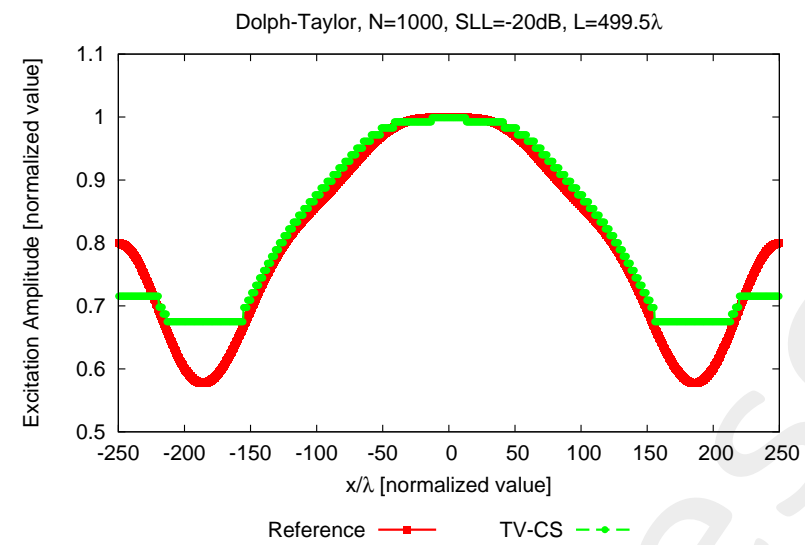

(a)

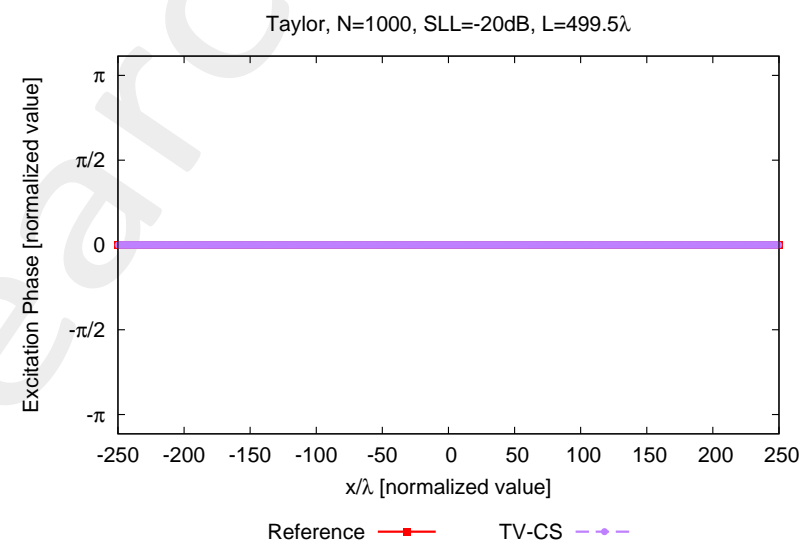

(b)

Figure 29: Performance Assessment (Taylor Pattern, $N=1000, S L L=-20 \mathrm{~dB}, d=0.5 \lambda, L=499.5 \lambda$, $C=65)$-Excitations amplitude (a) and phase (b).

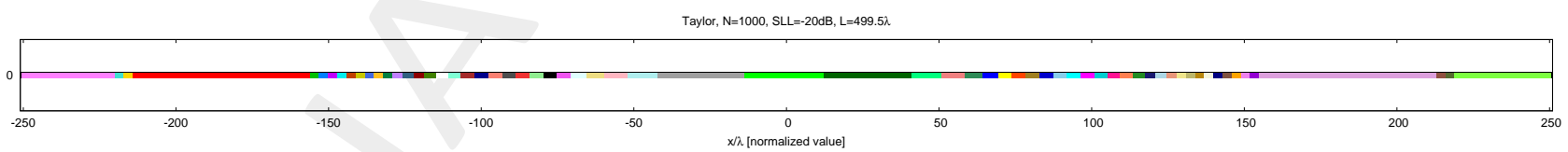

Figure 30: Performance Assessment (Taylor Pattern, $N=1000, S L L=-20 \mathrm{~dB}, d=0.5 \lambda, L=499.5 \lambda$, $C=65)$-Array elements clustering configuration.

\begin{tabular}{|c|c|c|c|c|c|c|}
\hline & $C$ & $S L L[\mathrm{~dB}]$ & $B W[\mathrm{deg}]$ & $D_{\max }[\mathrm{dB}]$ & $D R R_{\max }[\mathrm{dB}]$ & $\xi\left[\times 10^{-3}\right]$ \\
\hline \hline Reference & - & -19.87 & 0.1085 & 29.86 & 2.38 & - \\
\hline$T V-C S$ & 65 & -18.56 & 0.1084 & 29.90 & 1.7 & 2.14 \\
\hline
\end{tabular}

Table XII: Performance Assessment (Taylor Pattern, $N=1000, S L L=-20 \mathrm{~dB}, d=0.5 \lambda, L=499.5 \lambda$, $C=65)$ - Array Performance Indexes. 
Number of Clusters: $C=101$

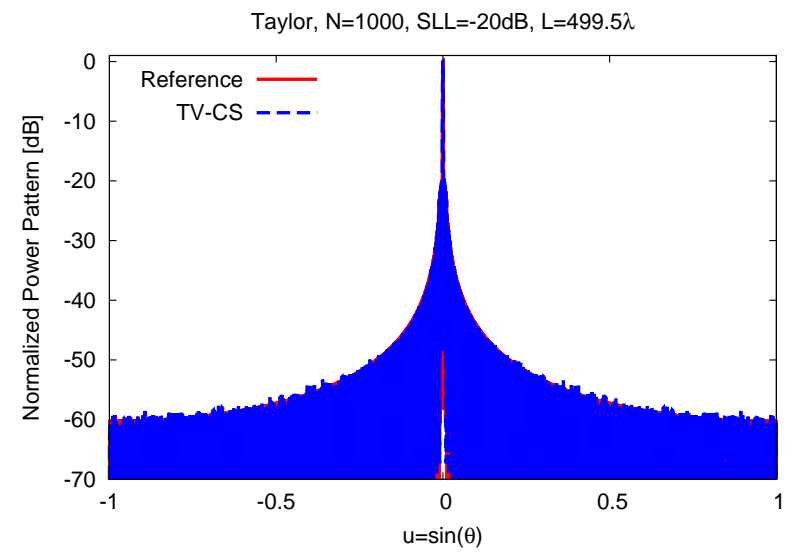

(a)

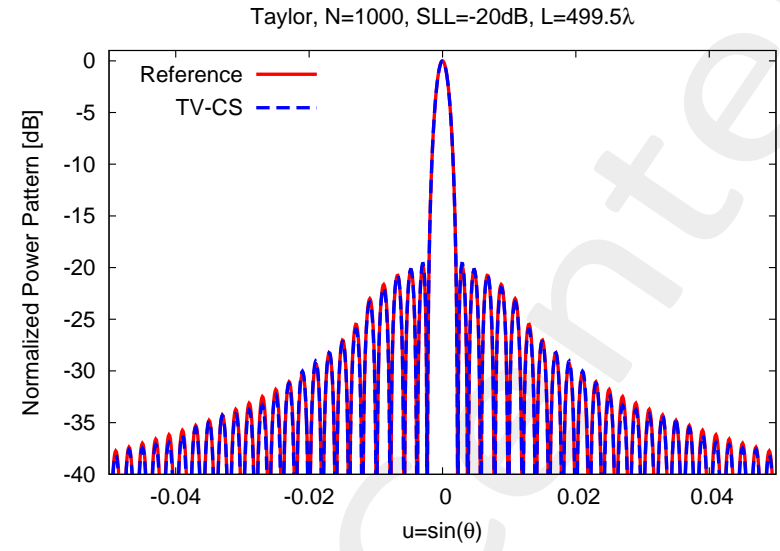

(b)

Figure 31: Performance Assessment (Taylor Pattern, $N=1000, S L L=-20 \mathrm{~dB}, d=0.5 \lambda, L=499.5 \lambda$, $C=101$ - -Power pattern over the whole visible $u$-range (a) and a detail of the main lobe (b).

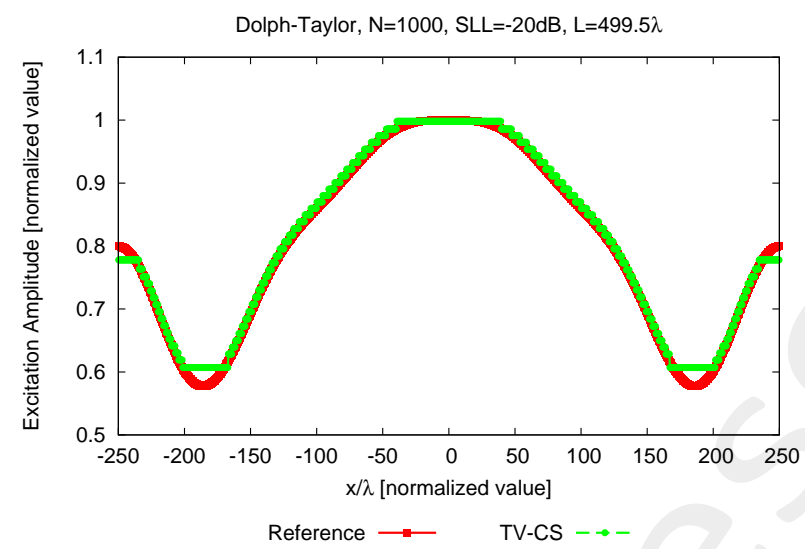

(a)

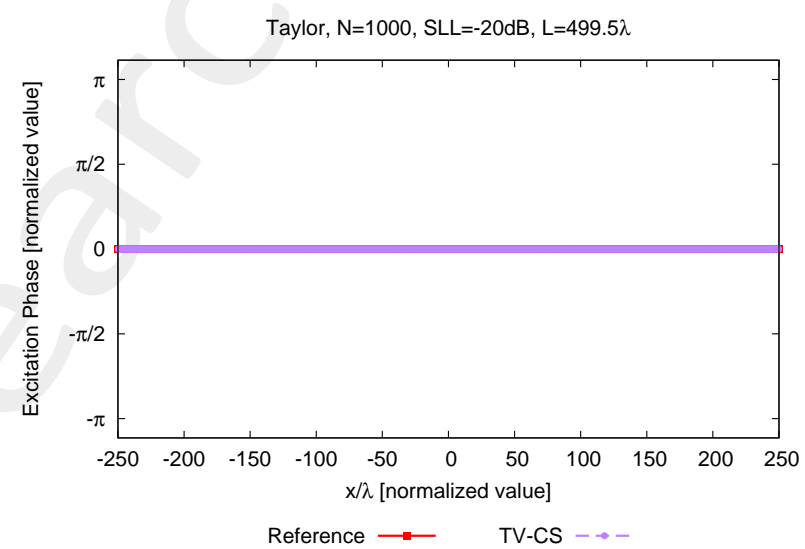

(b)

Figure 32: Performance Assessment (Taylor Pattern, $N=1000, S L L=-20 \mathrm{~dB}, d=0.5 \lambda, L=499.5 \lambda$, $C=101)$-Excitations amplitude (a) and phase (b).

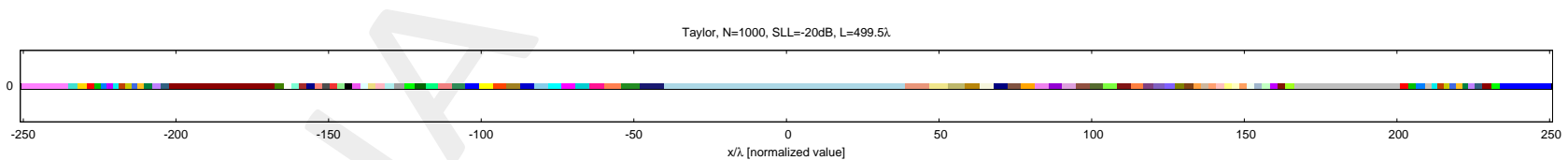

Figure 33: Performance Assessment (Taylor Pattern, $N=1000, S L L=-20 \mathrm{~dB}, d=0.5 \lambda, L=499.5 \lambda$, $C=101)$-Array elements clustering configuration.

\begin{tabular}{|c|c|c|c|c|c|c|}
\hline & $C$ & $S L L[\mathrm{~dB}]$ & $B W[\mathrm{deg}]$ & $D_{\max }[\mathrm{dB}]$ & $D R R_{\max }[\mathrm{dB}]$ & $\xi\left[\times 10^{-4}\right]$ \\
\hline \hline Reference & - & -19.87 & 0.1085 & 29.86 & 2.38 & - \\
\hline$T V-C S$ & 101 & -19.62 & 0.1085 & 29.87 & 2.16 & 1.12 \\
\hline
\end{tabular}

Table XIII: Performance Assessment (Taylor Pattern, $N=1000, S L L=-20 \mathrm{~dB}, d=0.5 \lambda, L=499.5 \lambda$, $C=101)$-Array Performance Indexes. 


\section{References}

[1] P. Rocca, G. Oliveri, R. J. Mailloux, and A. Massa, "Unconventional phased array architectures and design methodologies - A Review," Proc. IEEE, vol. 104, no. 3, pp. 544-560, Mar. 2016.

[2] G. Oliveri, G. Gottardi, F. Robol, A. Polo, L. Poli, M. Salucci, M. Chuan, C. Massagrande, P. Vinetti, M. Mattivi, R. Lombardi, and A. Massa, "Co-design of unconventional array architectures and antenna elements for $5 G$ base stations," IEEE Trans. Antennas Propag., vol. 65, no. 12, pp. 6752-6767, Dec. 2017.

[3] N. Anselmi, P. Rocca, M. Salucci, and A. Massa, "Irregular phased array tiling by means of analytic schemata-driven optimization," IEEE Trans. Antennas Propag., vol. 65, no. 9, pp. 4495-4510, Sep. 2017.

[4] G. Oliveri, "Multi-beam antenna arrays with common sub-array layouts," IEEE Antennas Wireless Propag. Lett., vol. 9, pp. 1190-1193, 2010.

[5] P. Rocca, R. Haupt, and A. Massa, "Sidelobe reduction through element phase control in sub-arrayed array antennas," IEEE Antennas Wireless Propag. Lett., vol. 8, pp. 437-440, 2009.

[6] P. Rocca, L. Manica, R. Azaro, and A. Massa, "A hybrid approach for the synthesis of sub-arrayed monopulse linear arrays," IEEE Trans. Antennas Propag., vol. 57, no. 1, pp. 280-283, Jan. 2009.

[7] G. Oliveri, P. Rocca, and A. Massa, "Reliable diagnosis of large linear arrays - a Bayesian compressive sensing approach," IEEE Trans. Antennas Propag., vol. 60, no. 10, pp. 4627-4636, Oct. 2012.

[8] A. Massa, P. Rocca, and G. Oliveri, "Compressive sensing in electromagnetics - A review," IEEE Antennas Propag. Mag., pp. 224-238, vol. 57, no. 1, Feb. 2015.

[9] G. Oliveri, M. Salucci, N. Anselmi, and A. Massa, "Compressive sensing as applied to inverse problems for imaging: theory, applications, current trends, and open challenges," IEEE Antennas Propag. Mag., vol. 59, no. 5, pp. 34-46, Oct. 2017.

[10] P. Rocca, M. A. Hannan, M. Salucci, and A. Massa, "Single-snapshot DoA estimation in array antennas with mutual coupling through a multi-scaling Bayesian compressive sensing strategy," IEEE Trans. Antennas Propag., vol. 65, no. 6, pp. 3203-3213, Jun. 2017.

[11] L. Poli, G. Oliveri, P. Rocca, M. Salucci, and A. Massa, "Long-distance WPT unconventional arrays synthesis," J. Electromagn. Waves Appl., vol. 31, no. 14, pp. 1399-1420, Jul. 2017.

[12] G. Oliveri, M. Salucci, and A. Massa, "Synthesis of modular contiguously clustered linear arrays through a sparseness-regularized solver," IEEE Trans. Antennas Propag., vol. 64, no. 10, pp. 4277-4287, Oct. 2016.

[13] F. Viani, G. Oliveri, and A. Massa, "Compressive sensing pattern matching techniques for synthesizing planar sparse arrays," IEEE Trans. Antennas Propag., vol. 61, no. 9, pp. 4577-4587, Sept. 2013.

[14] G. Oliveri and A. Massa, "Bayesian compressive sampling for pattern synthesis with maximally sparse non-uniform linear arrays," IEEE Trans. Antennas Propag., vol. 59, no. 2, pp. 467-481, Feb. 2011. 
[15] N. Anselmi, G. Oliveri, M. A. Hannan, M. Salucci, and A. Massa, "Color compressive sensing imaging of arbitrary-shaped scatterers," IEEE Trans. Microw. Theory Techn., vol. 65, no. 6, pp. 1986-1999, Jun. 2017.

[16] N. Anselmi, G. Oliveri, M. Salucci, and A. Massa, "Wavelet-based compressive imaging of sparse targets," IEEE Trans. Antennas Propag., vol. 63, no. 11, pp. 4889-4900, Nov. 2015.

[17] G. Oliveri, N. Anselmi, and A. Massa, "Compressive sensing imaging of non-sparse 2D scatterers by a total-variation approach within the Born approximation," IEEE Trans. Antennas Propag., vol. 62, no. 10, pp. 5157-5170, Oct. 2014.

[18] N. Anselmi, G. Gottardi, G. Oliveri, and A. Massa, "A total-variation sparseness-promoting method for the synthesis of contiguously clustered linear architectures" IEEE Trans. Antennas Propag., vol. 67, no. 7, pp. 4589-4601, Jul. 2019.

[19] M. Salucci, A. Gelmini, G. Oliveri, and A. Massa, "Planar arrays diagnosis by means of an advanced Bayesian compressive processing," IEEE Tran. Antennas Propag., vol. 66, no. 11, pp. 5892-5906, Nov. 2018. 